\title{
An Ultrasonic Sensor Based Low-Power Acoustic Modem for Underwater Communication in Underwater Wireless Sensor Networks
}

\author{
Heungwoo Nam and Sunshin An \\ Computer Network Lab., Dept. of Electronics Engineering, Korea University \\ 1, 5-Ga, Anam-dong, Sungbuk-gu, Seoul, Korea, Post Code: 136-701 \\ $\{$ hwnam, sunshin\}@dsys.korea.ac.kr
}

\begin{abstract}
Applications of underwater sensor networks involve environmental monitoring, disaster prevention, and resource detection. As the importance of these applications has recently grown, underwater sensor networks made up of sensor nodes have to be further investigated. However, little research has been performed to develop an underwater sensor node with communication functionality. In an underwater environment, typical RF-based communication is not appropriate because of two facts. One fact is that radio waves require large antennae and high transmission power. The other fact is that the Berkeley Mica 2 Motes have been reported to have a transmission range of $120 \mathrm{~cm}$ underwater. Consequently, we have concluded that underwater communication has to use an acoustic or ultrasonic wave rather than a radio wave. The objective of our work is to develop an acoustic modem for underwater communication, where we have to consider an energy-aware acoustic modem. This is because the battery can not easily be recharged underwater as well as in a terrestrial environment. As a consequence, an acoustic modem has to be designed with low-power. In this paper, we describe our implementation of an energy-aware acoustic modem and its performance in underwater experiments. ${ }^{1}$
\end{abstract}

Keywords: Underwater communication, Acoustic modem.

\section{Introduction}

Recent advances in processors, memory, and radio technology have made smart, tiny, and cheap nodes possible. Wireless sensor networks composed of these nodes can be used in promising network architectures. These sensors also collect a number of useful information in an unattended manner. Applications of underwater sensor networks are composed of environmental monitoring, disaster prevention,

\footnotetext{
${ }^{1}$ This research was supported by the MIC (Ministry of Information and Communication), Korea, under the 2007 ITRC (Information Technology Research Center) support program supervised by the IITA (Institute of Information Technology Assessment).
} 
and resource detection. As the importance of these applications has recently grown, underwater sensor networks made up of sensor nodes have to be further investigated. However, little research has been conducted in developing the underwater sensor node with communication functionality.

In an underwater environment, typical RF-based communication is not appropriate because of two facts. The first fact is that radio waves require large antennae and high transmission power. The other fact is that the Berkeley Mica 2 Motes, the most popular experimental platform, have been reported to have a transmission range of $120 \mathrm{~cm}$ in underwater at $433 \mathrm{MHz}$ [1]. Consequently, we have concluded that underwater communication has to use an acoustic or ultrasonic wave rather than a radio wave.

The objective of our work is to develop an acoustic modem for underwater communication, where we have to consider an energy-aware acoustic modem. That is because the battery can not be recharged underwater as well as in a terrestrial environment. As a consequence, the acoustic modem has to be designed with low-power. In this study, we implemented the energy-aware acoustic modem.

The contributions of this paper are as follows. First, this work develops a low-power based acoustic modem. In particular, our modem operates with a $3.3 \mathrm{~V}$ power supply. To that end, the lifetime of a sensor node was prolonged. Second, the modem for our study is a low-cost based acoustic modem with the capability for digital data communication. Because there is no existing modem with this capability based on low-cost, our modem is significant in this respect. Third, our work provides the basis for a ubiquitous device underwater as well as in a terrestrial environment. That is, the modem is smarter, smaller, and cheaper for underwater as well as terrestrial uses. As a result, this work's results are significant, including the result of having a technical method to perform real-time underwater monitoring effectively.

The remainder of this paper is organized as follows. Section 2 addresses related work on acoustic modems. In Section 3, we describe design principles and a hardware implementation of an acoustic modem. Section 4 describes underwater communication using software. The performance evaluation via underwater experiment is presented in Section 5. The comparisons and design challenges are provided in Section [6. In Section 7 we give some conclusions and future work.

\section{Related Work}

Acoustic communication plays an important role in underwater applications such as environmental monitoring, disaster prevention, and resource detection. Currently, underwater acoustic communication has received significant attention from many researchers. Previously, existing acoustic modems such as [2] 3] are commercial acoustic modems. However, they are usually bulky and expensive. They are not designed with low-power and they consume large amounts of 
energy. From this fact, we concluded that long-lived sensor networks over batterypowered nodes could not be implemented. In our state-of-the-art design, we focus on a low-power and low-cost acoustic modem.

There are acoustic modems designed with low-power. The authors in [4] proposed CORAL. It uses special acoustic hardware which consists of piezotransducers, a microcontroller-based architecture and interface circuitry. However, the difference between CORAL and our modem is the power supply. While the power supply of CORAL is $5 \mathrm{~V}$, the power supply of our modem is $3.3 \mathrm{~V}$. The authors in [5] proposed a Mica2 Sensor Board with an integrated Sounder and Microphone. In fact, their power supply in this system is similar to that in our modem. However, this system could not be used in underwater communication. The authors in [7] proposed a low-power acoustic modem. However, the power supply of this modem is $5 \mathrm{~V}$. Also, this modem does not perform underwater testing.

Different from the above, our modem uses ultrasonic sensors and its power supply is $3.3 \mathrm{~V}$. Also by taking the underwater test, it proves, itself, with underwater usage.

\section{A Hardware Implementation of an Acoustic Modem}

First, we now discuss the design principles of an acoustic modem. Our modem design includes several principles as follows. These principles are used to perform underwater communication effectively. i) Low-power based modem: Sensor networks composed of sensor nodes are based on a low-power design. Batteries can not be recharged underwater. Thus, to allow the long-lived operation of sensor nodes, we have to design a low-power modem. ii) Low-cost based modem: In our study, we found commercial acoustic modems usually bulky and expensive. We concluded that commercial acoustic modems did not allow for much wider deployment. Also, devices such as a submarine and an autonomous underwater vehicle (AUV) used acoustic communication, but were expensive. We, therefore, have to make a low-cost modem for wider dissemination. iii) Modem with the capability for digital data communication: Most underwater communications has been accomplished by sending only analog data such as voice. There is no underwater communication using a microcontroller which processes digital data. That is why many researchers investigate underwater communications sending digital data. Hence, by this fact, we have to develop an acoustic modem to communicate digital data by means of a microcontroller.

This work implements an underwater acoustic modem according to the above design principles. The microcontroller for the sensor node is an ATmega128L, which has an 8bit MCU, and operates from a 3.3 volt power supply. Hence, the power supply of our underwater modem is also 3.3 volt.

Fig. 1 shows a block diagram of an acoustic modem. Our acoustic modem is divided into two main parts: an ultrasonic transmitter and an ultrasonic receiver. These parts used ultrasonic sensors to generate or detect the ultrasonic wave. The ultrasonic sensors, which are waterproof, use a $40 \mathrm{kHz}$ frequency both for 


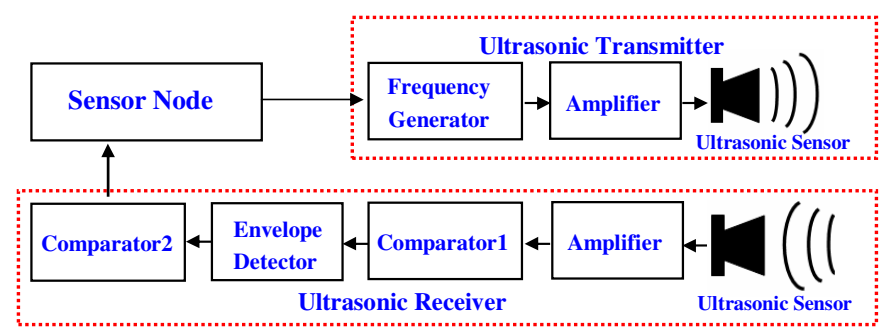

Fig. 1. A block diagram of an acoustic modem

the transmitter sensor and the receiver sensor. We, next, describe the details of this ultrasonic transmitter and receiver.

\subsection{Ultrasonic Transmitter and Receiver}

The ultrasonic transmitter consists of three components: a frequency generator, an amplifier, and an ultrasonic sensor. The frequency generator makes a $40 \mathrm{kHz}$ frequency and uses an ASK modulation/demodulation method that combined an on-off voltage level with a carrier wave. Then, the modulated signals in the frequency generator are sent to the amplifier. The amplifier amplifies these input signals. The voltage level of the amplified signals is $-6 \mathrm{~V} \sim+6 \mathrm{~V}$. Finally, the ultrasonic sensor transmits these amplified signals.

The ultrasonic receiver is composed of five components: an ultrasonic sensor, an amplifier, an envelope detector, and two comparators. The ultrasonic sensor receives the transmitted signals and sends them to the amplifier. Because the received signals are a minute signal, to process them in the next step, they have to be amplified. So, the amplifier amplifies the received signals. Then, the comparator 1 removes the noise from the amplified signals and sends the signals to the envelope detector. The envelope detector detects the original signals from the amplified signals. Finally, the comparator 2 transforms these analog signals into digital signals and sends them to the sensor node. The sensor node uses software to regenerate the transmitted data.

\section{Underwater Communication Software}

\subsection{Packet Format}

We use a packet which is composed of two flags, a payload header and a payload. Here, two flags carry out the functionality of an acoustic physical layer header. A payload header performs the functionality of an acoustic MAC layer header. That is why we are able to make up the compact packet and carry out the test.

Fig. 2 shows our packet format. For more explanation, we describe all the elements of the packet. 


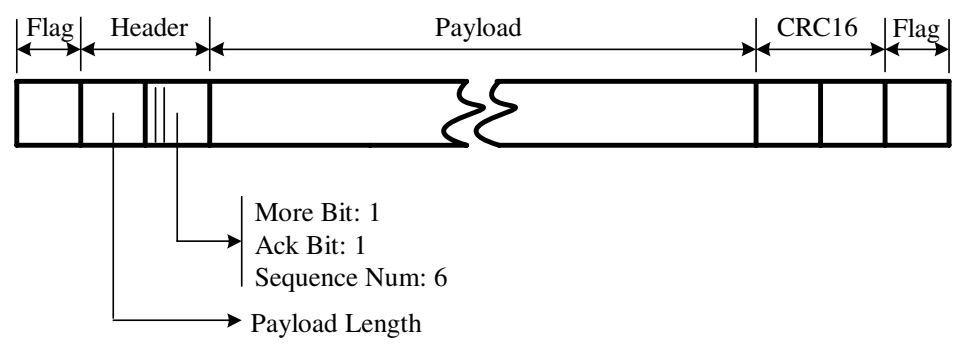

Fig. 2. The packet format

- Flag: The flag uses a bit sequence of 11011011. It is used to indicate the start and end of a packet. The length of the flag is 1 byte. The start bit of the flag is 1 . This makes a sensor node know that a packet arrives. So, the sensor node generates an interrupt to perform a software sampling method which is provided in Subsection 4.4 .

- Payload length: The payload length is the size of the payload. It has 1 byte.

- More bit: The more bit is used to recognize the end of the packet. Its length is 1 bit.

- Ack bit: The ack bit is used to distinguish between the data packet and the ack packet. The length of the ack bit is 1 bit.

- Sequence number bit (SNB): The sequence number bit shows the order of each packet. It has 6 bits.

- CRC (Cyclic Redundancy Check): The CRC is at end of the packet. In our implementation, we use CRC16, which has 2 bytes, to detect the transmitting errors. The value of CRC16 is extracted from CRC16-Table which is made up of 256 elements.

The length of the payload is not fixed. Its length is decided according to the kind of applications. This work uses a 20 bytes payload to carry out the underwater communication. In this experiment, we do not consider a number of MAC services. These MAC services will be left for future work.

\subsection{The Transmitted Signals in the Ultrasonic Transmitter}

The modulation method used in this work is an ASK. The ASK identifies whether the signal is sent or not, and generates the signal according to this fact. For example, when transmitting the bit '1', the frequency generator generates 40 $\mathrm{kHz}$ during $t$ time. Otherwise, it does not generate the frequency during $t$ time. That is, the frequency generator uses an on/off method according to the data from the MCU and transmits the data. For more explanation we provide Fig. 3. The amplifier uses the RS232 IC to amplify the signals which come from the frequency generator. Fig. 3 shows these amplified signals. 

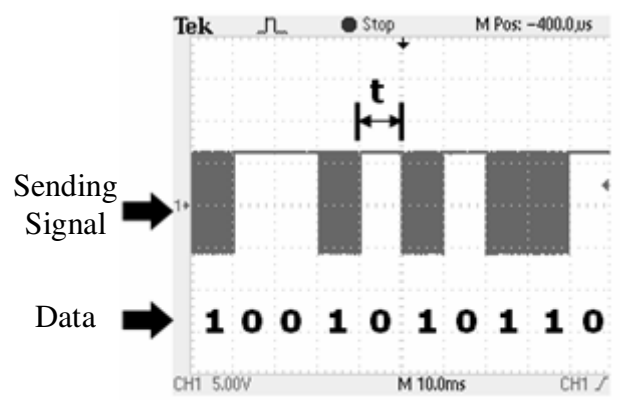

Fig. 3. The signals in the amplifier

Here, we define the sending period, $t$ time (ms). This time is defined as the time interval of transmitting each bit. Thus the data rate is denoted in Formula (1):

$$
\text { DataRate }=1000 / t
$$

If the performance of the ultrasonic sensor is high, the data rate will be improved much more. This will be explained in Section 5 .

\subsection{The Received Signals in the Ultrasonic Receiver}

The ultrasonic sensor receives the transmitted signals and sends them to the amplifier. Because the received signals are a minute signal, they have to be amplified. Fig. 4(a) shows the amplified signal in the amplifier. Then, the comparator1 removes the noise. The envelope detector detects the original signals from the amplified signals. Finally, the comparator2 transforms these analog signals into digital signals and sends them to the sensor node. The demodulated signal, later to be transmitted to the sensor node, in the comparotor 2 is shown

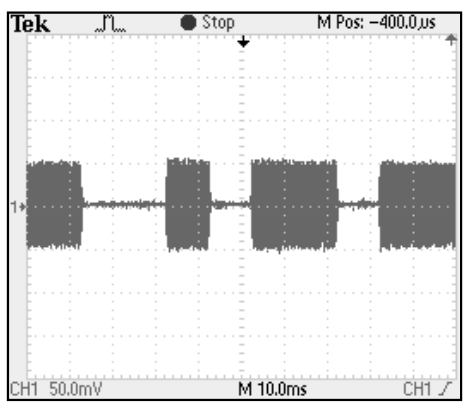

(a) The amplified signals

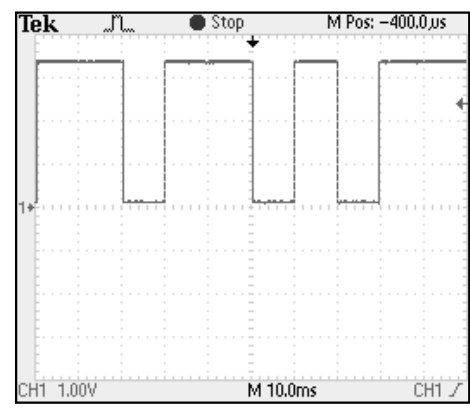

(b) The signals in the comparator2

Fig. 4. The signals in the receiver part 
in Fig. 4(b). These signals are entered into the comparator port in the sensor node. The sensor node uses software to retrieve the original data.

\subsection{The Software Sampling Method}

The signals, which are shown in Fig. 四(b), are entered into the comparator port in the sensor node. The comparator port uses a software sampling method to interpret these signals as the origianl signals. The software sampling method is as follows. The comparator port generates an interrupt at an interval of $t / 2$ time from the high toggled signal to read the entered signal. Next, the port reads the entered signal at regular intervals of $t$ time. For a more detailed explanation, we provide Fig. 5 which shows the software sampling method. Thus, if the value of the signal is greater than the value of the comparator, the comparator port represents the signal as 1 . Otherwise, the port identifies it as 0 .

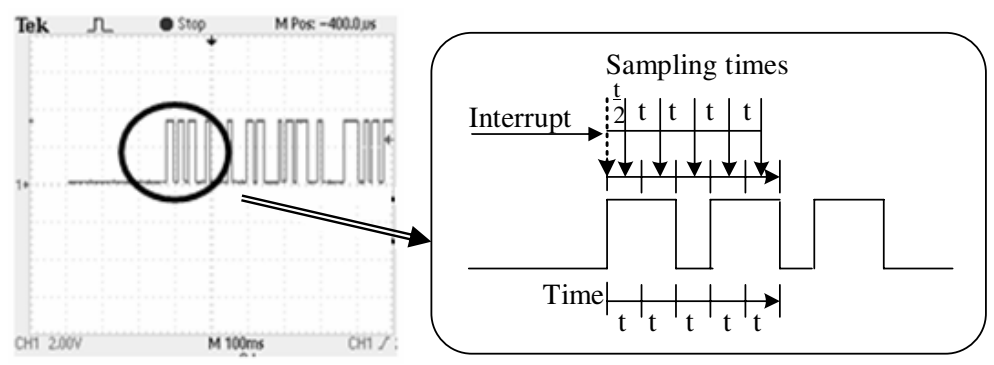

Fig. 5. The software sampling method

\section{Performance Evaluation}

\subsection{Test Environment for Underwater Communication}

We used an aquarium to perform underwater communication. Fig. [6 shows the test environment. Fig. 6(a) is a picture of the overall system. For more explanation, we illustrate with Fig. 6(b). Two notebooks were connected with two sensor nodes which were linked to the acoustic modems in the water. The sensor node used the ATmega128L MCU. In the near future, these sensor nodes will be put in the water. The notebook used the UART to communicate with the sensor node. The UI was a hyper-terminal. The underwater communication between the sensor nodes was performed by sending text.

The communication experiment was conducted with the following steps. First, in the transmitter part, we inputted the data to be transmitted in the notebook. Then, the sensor node sent the data to the acoustic modem. The acoustic modem transformed the data into an analog signal and transmitted it through the ultrasonic sensor. In another receiver part, the acoustic modem amplified the 


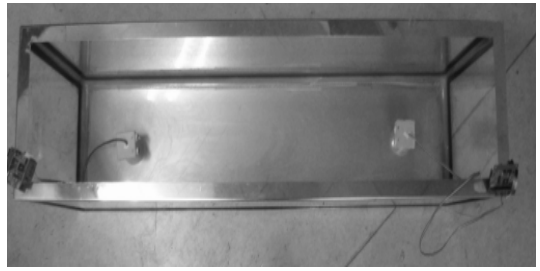

(a) The picture

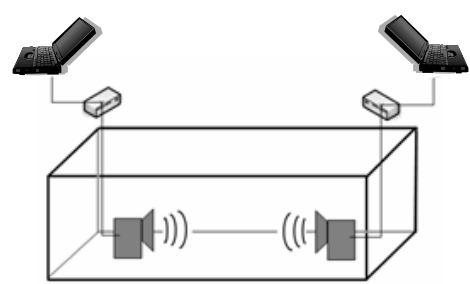

(b) The overall schematic

Fig. 6. The test environment

signals received by the ultrasonic sensor and transformed the amplified signals into digital signals. Then, the sensor node interpreted the digital signals and sent them to the notebook. Finally, we identified the transmitted data in the notebook.

\subsection{The Sending Period and Communication Distance}

Based on our test environment, we carried out the experiments by changing the sending periods. In our experiments, the length of the payload was set to 20 bytes. For each sending period, the transmitter sent 100 packets to the receiver. The receiver recorded the number of the packets received correctly to compute the packet delivery ratio.

Fig. 7 shows the different packet deliver ratios when the sending period changed. As shown in the figure, the packet delivery ratio increased as the sending period increased. For a 10ms sending period, we obtained a packet deliver

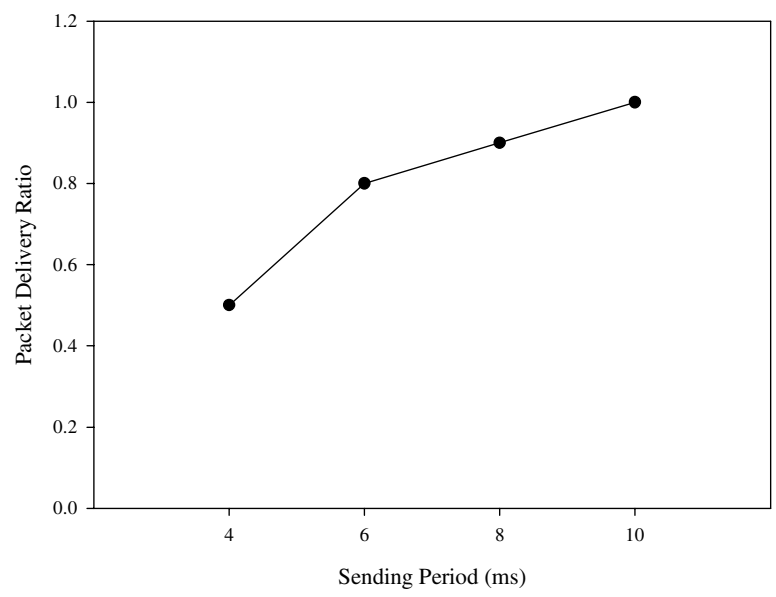

Fig. 7. Packet delivery ratio vs. the sending period 
ratio 1 . This means that the packet deliver error is not generated. So, we define the data rate of our modem as 100 bps. If the acoustic modem can be improved in the ultrasonic sensors, we can decrease the sending period to increase the data rate. This remains for future work.

We did not consider the communication distance. That was because we focused on whether our modem operated very well or not. Fortunately, the communication distance of our modem was $3 \mathrm{~m}$. Therefore, finding the exact range of the acoustic modem would have required an enlarged test facility.

\section{The Comparisons and Design Challenges}

To evaluate the performance of our acoustic modem, we provide Table 1. Table 1 has the evaluations of and comparisons of the performance of the acoustic modems. Because these modems were very recently developed with low-power and low-cost, we compared them so that we could represent the superiority of our modem from the performance perspective.

The comparisons of the acoustic modems are as follows. First, the power supply of our modem was $3.3 \mathrm{~V}$, and all the others had a $5 \mathrm{~V}$ power supply. Here, we did not know the power supply of the acoustic communication [5]. So, we inferred its power supply from the usage of the sounder and microphone as used in lowpower acoustic modem [7. In the energy consumption perspective, our modem was the best of all the others. Second, while our modem and the CORAL 4 . were used in the underwater experiment, the Acoustic Communication [5] and the Low-Power Acoustic Modem [7] were not performed in the underwater test. Also, while the test of CORAL 4 put only sensors in the water, our test involved testing the acoustic modem and sensors in the water. From an objective point of view as to the number of devices in the water, our test was much more concerned with the underwater experiment. Third, while the data rate of our modem was $100 \mathrm{bps}$, the data rate of the Acoustic Communication [5] was less than 10bps. By this fact, the data rate of our modem was ten times as fast as that of the Acoustic Communication [5. We did not know the data rate of the CORAL 4] and the Low-Power Acoustic Modem [7. From the viewpoint of the data rate, our modem had the best performance. Fourth, the frequencies of the CORAL [4, the Acoustic Communication [5], the Low-Power Acoustic Modem [7], and our modem were $1.7 \mathrm{kHz}, 4.5 \mathrm{kHz}, 18 \mathrm{kHz}$, and $40 \mathrm{kHz}$ respectively. Finally, the transmitting/receiving devices of the CORAL [4, the Acoustic Communication [5, the Low-Power Acoustic Modem [7, and our modem were the piezo-transducers, the sounder and microphone, the projector/speaker and hydrophone/microphone, and the ultrasonic sensors respectively. So, only our modem used the ultrasonic sensors. As the above comparison indicated, we concluded that the functionality of our acoustic modem came significantly forward in the power supply, the underwater experiment, and the improved data rate aspects.

The design challenges to overcome for the underwater modem are as follows. i) The directional property: The directional property of the ultrasonic sensor is so 
Table 1. A comparisons of the acoustic modems

\begin{tabular}{|c|c|c|c|c|c|}
\hline $\begin{array}{l}\text { Acoustic } \\
\text { Modem }\end{array}$ & $\begin{array}{c}\text { Power Supply } \\
\text { (Microcontroller) }\end{array}$ & $\begin{array}{l}\text { Underwater } \\
\text { Experiment }\end{array}$ & $\begin{array}{l}\text { Data } \\
\text { Rate } \\
\text { (bps) }\end{array}$ & $\begin{array}{l}\text { Freq- } \\
\text { uency } \\
(\mathrm{kHz})\end{array}$ & $\begin{array}{c}\text { Transmitting } \\
\text { /receiving } \\
\text { Device }\end{array}$ \\
\hline CORAL 4 & $\begin{array}{c}5 \mathrm{~V} \\
(\mathrm{TI} \mathrm{MSP} 430)\end{array}$ & $\begin{array}{l}\text { O (only } \\
\text { sensors) }\end{array}$ & - & 1.7 & $\begin{array}{c}\text { Piezo- } \\
\text { transducers }\end{array}$ \\
\hline $\begin{array}{l}\text { Acoustic } \\
\text { Commun- } \\
\text { ication [5] }\end{array}$ & $\begin{array}{c}5 \mathrm{~V} \\
(\mathrm{MICA} 2 \text { Mote) }\end{array}$ & $X$ & $<10$ & 4.5 & $\begin{array}{c}\text { Sounder, } \\
\text { Microphone }\end{array}$ \\
\hline $\begin{array}{l}\text { Low-Power } \\
\text { Acoustic } \\
\text { Modem [7] }\end{array}$ & $\begin{array}{c}5 \mathrm{~V} \\
(\text { MICA2 Mote) }\end{array}$ & $\mathrm{X}$ & - & 18 & $\begin{array}{c}\text { Projector/ } \\
\text { Speaker, } \\
\text { Hydrophone/ } \\
\text { Microphone }\end{array}$ \\
\hline $\begin{array}{c}\text { Our acoustic } \\
\text { Modem }\end{array}$ & $\begin{array}{c}3.3 \mathrm{~V} \\
(\text { ATmega128L) }\end{array}$ & $\begin{array}{c}\mathrm{O} \text { (acoustic } \\
\text { modem and } \\
\text { sensors) }\end{array}$ & 100 & 40 & $\begin{array}{c}\text { Ultrasonic } \\
\text { Sensors }\end{array}$ \\
\hline
\end{tabular}

sensitive that if the direction is not matched, its transmitting error is increased. To that end, the pinpoint development of an ultrasonic sensor is needed. The underwater acoustic modem also has to process a minute signal. ii) The reflection and refraction: The reflection and refraction of a signal in underwater are very hard. That is why underwater communication is more difficult than terrestrial communication. The underwater acoustic modem has to remove the noise such as the reflection and refraction.

\section{Conclusion and Future Work}

Through related work, we knew the requirement that the underwater sensor networks must perform the acoustic communication. According to this requirement, this research with regard to an acoustic modem has significant meaning when performing acoustic communication. However, the hardware to support acoustic communication did not exist at all prior to our work.

This work developed an acoustic modem as hardware to perform acoustic communication. Thus, the advantages of our acoustic modem are as follows. First, our acoustic modem is a low- powered acoustic modem. In the energy consumption perspective, our modem was the best of all the others. Second, our modem is a low-cost based acoustic modem with the capability of digital data communication. Because there had been no prior existing modem with this 
capability based on low-cost, our modem is significant in this regard. Through Table 1, we do know the fact that our modem come to the fore among the acoustic modems which have developed recently.

Some of the problems to be considered in our acoustic modem are: the directional property, the reflection, and the refraction. Our future work is the research to overcome these problems. Because the underwater applications have significant attraction of themselves, the acoustic modem with the functionality of the underwater communication will become much more significant. Therefore, it is our task to develop the acoustic modem to its fullest potential. In addition, this acoustic modem will become the basis for the underwater wireless sensor networks.

\section{References}

1. Akyildiz, I.F., Pompili, D., Melodia, T.: Underwater Acoustic Sensor Networks: Research Challenges. Elsevier's Journal of Ad Hoc Networks 3(3), 257-281 (2005)

2. Benthos, Inc., Fast And Reliable Access To Undersea Data, http://www.benthos.com/pdf/Modems/ModemBrochure.pdf

3. LinkQuest, Inc., Underwater Acoustic Modems, http://www.link-quest.com/html/uwm_hr.pdf

4. ya, Engel, J., Chen, J., Fan, Z., Liu, C.: CORAL: Miniature Acoustic Communication Subsystem Architecture for Underwater Wireless Sensor Networks. In: The 4th IEEE International Conference on Sensors (October 2005)

5. Zhang, J., Huang, Z., Liu, X.: Acoustic Communication in Wireless Sensor Networks. In: CS651, Wireless Sensor Networks, pp. 1-8 (December 2005)

6. Crossbow Technologies, Inc., http://www.xbow.com/

7. Wills, J., Ye, W., Heidemann, J.: Low-Power Acoustic Modem for Dense Underwater Sensor Networks. In: Proceedings of the First ACM International Workshop on Underwater Networks (WUWNet), pp. 79-85 (September 2006) 